Photogallery

\title{
Bail-out of the polyp from the skeleton of spats in the scleractinian coral Acropora tenuis
}

\section{Shiho T. KARIYAZONO ${ }^{1}$ and Masayuki HATTA ${ }^{2, *}$}

${ }^{1}$ Department of Evolutionary Studies of Biosystems, the Graduate University for Advanced Studies, Hayama, Kanagawa 240-0193, Japan

${ }^{2}$ Department of Biology, Ochanomizu University, Tokyo 112-8610, Japan

* Corresponding author: Masayuk Hatta

E-mail: hatta.masayuki@ocha.ac.jp

Communicated by Michio Hidaka (Editorial Board Member)

Keywords Acropora, Bail-out, Coral, Skeleton, Polyp

Unlike tight association between the polyp and skeleton in scleractinian corals, there are reports on bail-out of polyps from the colony in Seriatopora (Sammarco 1982), expulsion of polyp with calices in Favia and Oculina (Kramarsky-Winter et al. 1997), and also reversal to a motile palnula form from the initial polyp in Pocillopora (Richmond 1985). Here we report polyp bail-out from spats in Acropora. Hundreds of primary polyps of Acropora tenuis were prepared in culture dishes following Iwao et al. (2002) from gametes collected in the field, infected with zooxanthellae from the giant clam and reared at $25^{\circ} \mathrm{C}$ with daily seawater change. Two months later, we found more than a dozen of naked polyps floating in the culture dishes (Fig. 1A). They were active for more than 4 weeks, and some looked healthy with extended tentacles and buds (Fig. 1B).
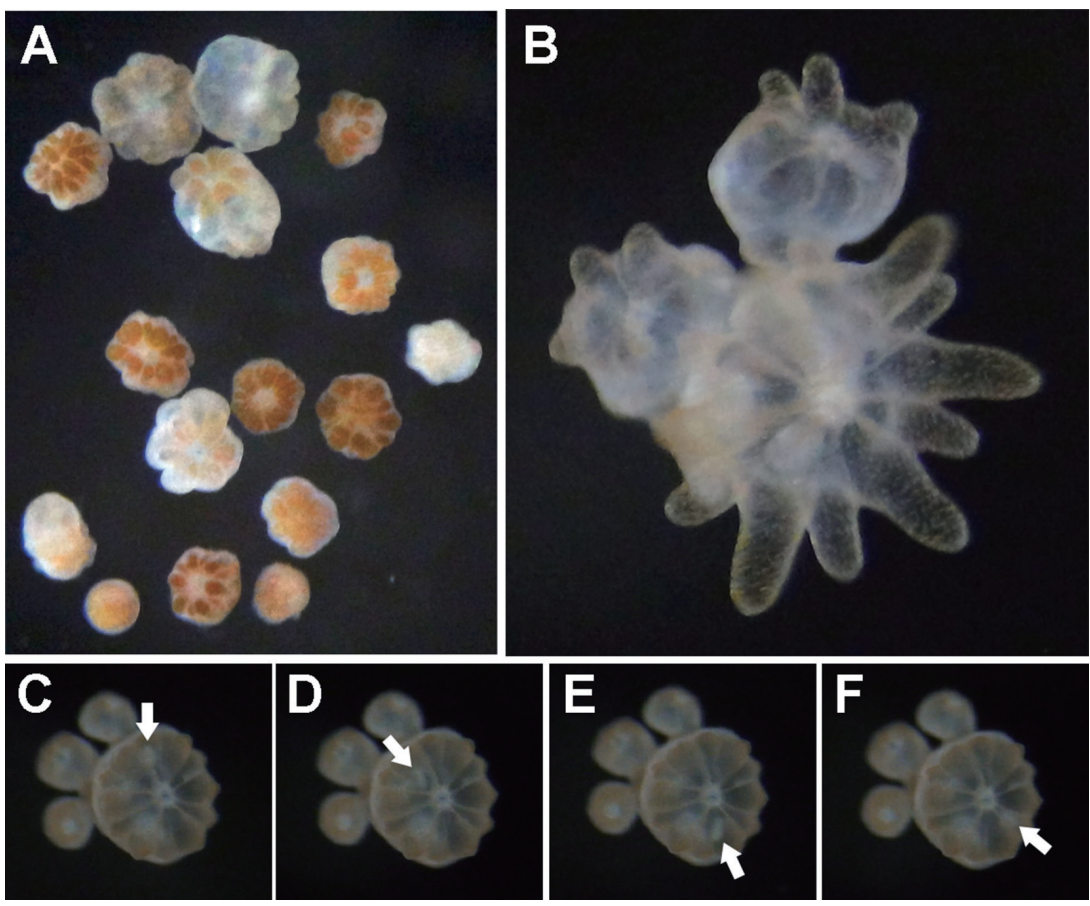

Fig. 1 Polyps bailed out from the spat skeleton. A A snap shot of polyps bailed out. Different tones of brown colors reflect the density of zooxanthellae. B A bailed-out polyp possessing two buds, extending tentacles. $\mathbf{C}-\mathbf{F}$ A bailed-out polyp with three buds. An arrow indicates a white particle circulating in the entire polyp. 
Although it remains mysterious how polyps with buds bailed out from the reticulate skeletal complexes in the coenosteum region, these observations reveal that the polyp of Acropora is capable of bailing out from, and of surviving without skeletons. In addition, circulation of particles in the whole body of bailed-out polyp (Fig. 1C-F) shows loss of the compartment structure observed in the primary polyp of Acropora (Hatta 2010). It suggests that the compartment structure is maintained in a simple cylindrical body by partitioning skeletons.

\section{Acknowledgments}

We thank Akajima Marine Science Laboratory for sampling supports.

\section{References}

Hatta M (2010) Unidirectional circulation in each coelenteron compartment in a primary polyp of Acropora tenuis. Galaxea, JCRS 12: 45

Iwao K, Fujisawa T, Hatta M (2002) A cnidarian neuropeptide of the GLWamide family induces metamorphosis of reefbuilding corals in the genus Acropora. Coral Reefs 21: 127-129

Kramarsky-Winter E, Fine M, Loya Y (1997) Coral polyp expulsion. Nature 387: 137

Richmond RH (1985) Reversible metamorphosis in coral planula larvae. Mar Ecol Prog Ser 22: 181-185

Sammarco WP (1982) Polyp bail-out: an escape response to environmental stress and a new means of reproduction in corals. Mar Ecol Prog Ser 10: 57-65

Received: 30 October 2013/Accepted: 17 April 2015

(C) Japanese Coral Reef Society 